\title{
National treatment trends, complications, and predictors of in-hospital charges for the surgical management of craniopharyngiomas in adults from 2007 to 2011
}

\author{
Hasan A. Zaidi, M.D., Kristina Chapple, Ph.D., and Andrew S. Little, M.D. \\ Division of Neurological Surgery, Barrow Neurological Institute, St. Joseph's Hospital and Medical Center, \\ Phoenix, Arizona
}

Object. Treatment of craniopharyngiomas is one of the most demanding and controversial neurosurgical procedures performed. The authors sought to determine the factors associated with hospital charges and fees for craniopharyngioma treatment to identify possible opportunities for improving the health care economics of inpatient care.

Methods. The authors analyzed the hospital discharge database of the Nationwide Inpatient Sample (NIS) covering the period from 2007 through 2011 to examine national treatment trends for adults (that is, those older than 18 years) who had undergone surgery for craniopharyngioma. To predict the drivers of in-hospital charges, a multistep regression model was developed that accounted for patient demographics, acuity measures, comorbidities, hospital characteristics, and complications.

Results. The analysis included 606 patients who underwent resection of craniopharyngioma; 353 resections involved a transsphenoidal approach (58\%) and 253 a transfrontal approach $(42 \%)$. The mean age $( \pm$ SD) of patients was $47.7 \pm 16.3$ years. The average hospital length of stay (LOS) was $7.6 \pm 9$ days. The mean hospital charge $( \pm$ SD) was $\$ 92,300 \pm \$ 83,356$. In total, $48 \%$ of the patients experienced postoperative diabetes insipidus or an electrolyte abnormality. A multivariate regression model demonstrated that LOS, hospital volume for the selected procedure, the surgical approach, postoperative complications, comorbidities, and year of surgery were all significant predictors of in-hospital charges. The statistical model accounted for $54 \%$ of the variance in in-hospital charge.

Conclusions. This analysis of inpatient hospital charges in patients undergoing craniopharyngioma surgery identified key drivers of charges in the perioperative period. Prospective studies designed to evaluate the long-term resource utilization in this complex patient population would be a useful future direction.

(http://thejns.org/doi/abs/10.3171/2014.8.FOCUS14366)

$\begin{array}{llll}\text { KEY WORDS } & \text { craniopharyngioma } & \bullet & \text { Nationwide Inpatient Sample } \\ \text { transsphenoidal } & \bullet & \text { parasellar } & \bullet\end{array}$

$\mathrm{C}$ RANIOPHARYNGIOMAS are locally aggressive intracranial tumors arising along the path of the craniopharyngeal duct. Surgical intervention is often the first step in their management, and it involves either a transsphenoidal or transfrontal approach to enable a tissue diagnosis and to debulk the tumor mass. ${ }^{4,6,17,18,24,28,31}$ Because craniopharyngioma are rare, surgical outcome data for patients with craniopharyngiomas are limited to small case series at academic centers, and national trends of treatments of the disease have not been examined.

Resource utilization after surgery has become a health care metric receiving increased scrutiny. This type of analysis requires a multicenter experience to examine the variables associated with caring for a diverse group of patients in the immediate perioperative period. Analyses in the literature of the drivers of hospital resource use in the management of spinal, $, 10,20,32,33$ cerebrovascular, ${ }^{13,14}$

\footnotetext{
Abbreviations used in this paper: $\mathrm{ED}=$ emergency department; HCUP $=$ Healthcare Cost and Utilization Project; LOS = length of stay; NIS = Nationwide Inpatient Sample.
}

and pituitary ${ }^{22}$ diseases have helped to identify those variables that can be modified to improve this use. Previous work suggests that complication rates, provider volumes, and surgical approaches all influence hospital charges. ${ }^{2,22}$ Here, we set out to examine contemporary national treatment trends, complications, and predictors of inpatient charges, using the Nationwide Inpatient Sample (NIS) for patients undergoing surgery for craniopharyngiomas.

\section{Methods}

\section{Data Collection}

All data for this analysis had been collected from 2007 through 2011 and obtained from the NIS Healthcare Cost and Utilization Project (HCUP) at the Agency for Healthcare Research and Quality (http://www.hcupus.ahrq.gov/nisoverview.jsp). The NIS discharge database approximates a 20\% sample of all inpatient admissions to nonfederal US hospitals from 44 states, including a total of 8 million inpatient hospital stays per year. 


\section{Inclusion and Exclusion Criteria}

The International Classification of Diseases, 9th Revision, Clinical Modification, or the ICD-9-CM, diagnostic and primary procedure codes were used to identify the patients to be included in our analysis. Patients with a primary diagnosis code of 237.0 (craniopharyngioma) were included. Patients younger than 18 years and those with a concomitant diagnosis code of 253.8 (other disorders of the pituitary and other syndromes of diencephalohypophyseal origin, excluding a diagnosis of craniopharyngioma) were excluded from our study, as were all nonoperative cases. Only those patients who underwent surgical intervention for a craniopharyngioma were included. The surgical approach was identified as a categorical variable, including "transsphenoidal" (codes 07.65 and 07.62) or "transfrontal" (codes 07.61 and 07.64). The "transfrontal" approach includes all transcranial approaches as designated in the NIS database. Cases with total in-hospital charges greater than 3 SDs above the mean were excluded from our final analysis to avoid biasing the results in favor of a few high-utilization cases.

\section{Cohort Characteristics}

Patient age (in years) and length of stay (LOS; in days) were modeled as continuous variables. Ethnicity was categorized as "white" versus "other." The payer source was also available in the NIS database for analysis and was categorized as private insurance, Medicare, Medicaid, self-pay, or other.

Postoperative complications were scored dichotomously ( 0 versus 1 or more complications) and also modeled individually. The latter was done to identify specific complications that had the greatest impact on in-hospital charges. On the basis of our previous findings for patients with Cushing disease, ${ }^{22}$ we hypothesized that complications would be statistically associated with increased inhospital charge. The complications included in the final analysis included the following: fluid and electrolyte abnormalities (codes 276.0-276.9) and diabetes insipidus (code 253.5) were compiled as a single entity; postoperative neurological complications including those due to infarction or hemorrhage (codes 997.00-997.09); deficits of cranial nerves (codes 368.2, 374.30-31, and 378.50-59); iatrogenic panhypopituitarism (code 253.7); cerebrospinal rhinorrhea (code 349.81); hematoma (998.1-998.13) or intracerebral hemorrhage event (code 430-432 or codes 998.1-998.13); pneumonia (code 486); deep venous thrombosis (codes 453.8-9 and 38.7); iatrogenic pulmonary embolus (codes 415.11-19); placement of inferior vena cava filter (code 38.93); myocardial infarction (code 410); blood transfusion (code 99.04); performance of angiography (code 88.41); postoperative infection (code 998.5); and renal failure (code 639.3).

Patient comorbidities were also dichotomized as none or 1 versus $\geq 2$. Comorbidities available for analysis in the NIS include alcohol abuse, deficiency anemia, rheumatoid arthritis, collagen vascular diseases, chronic blood loss anemia, congestive heart failure, chronic pulmonary disease, coagulopathy, depression, uncomplicated diabetes, diabetes associated with chronic complications, drug abuse, hypertension, hypothyroidism, liver disease, lymphoma, metastatic cancer, neurological disorders, obesity, paralysis, peripheral vascular disorders, psychoses, pulmonary circulation disorders, renal failure, solid tumor without metastasis, valvular disease, and weight loss.

\section{Hospital Characteristics}

The NIS database categorizes hospitals by the number of beds (dichotomized for our analysis as "small" versus "medium to large"), and teaching status of hospitals ("teaching" versus "nonteaching"). The definition in the NIS database of hospital size by the number of beds depends on geographic location and hospital type, and the specific cutoffs for this definition of hospital size are provided on the HCUP website. ${ }^{16}$ Individual hospitals were also categorized as low volume $(\leq 20$ procedures over a 5-year period) or high volume ( $>20$ procedures over a 5-year period), which is similar to the cutoff values used to dichotomize high- versus low-volume hospitals in previous studies analyzing administrative databases for transsphenoidal procedures..$^{2,25,26}$ For this study, "procedure" was defined as noted in the inclusion/exclusion criteria (adult patients with a primary diagnosis of craniopharyngioma undergoing transsphenoidal or transfrontal surgical approaches).

\section{Statistical Analysis}

Data were analyzed with the statistical software program SPSS (version 21), using descriptive statistics, Pearson correlations, chi-square analyses, ANOVA, and stepwise multivariate linear regression. Means \pm SDs are reported unless otherwise noted. Probability values presented were 2 tailed and considered significant at $<0.05$. Logarithmically transformed values for hospital charges and LOS were used in the regression model to account for skewness. As per the standard data use agreement with HCUP, variables with $\leq 10$ data points included are not reported in this study.

\section{Results}

\section{Patient Selection and Characteristics}

From 2007 through 2011, a total of 629 adults whose data were in the HCUP database had undergone either transsphenoidal surgery or craniotomy for resection of craniopharyngioma. We excluded 16 patients for whom total charge data were missing and 7 for whom in-hospital charges exceeded 3 SDs from the mean, leaving 606 cases for analysis. The average hospital charge was $\$ 92,300 \pm \$ 83,356$, with over half of the patients $(58 \%)$ undergoing transsphenoidal surgery. The average age of our cohort was $47.7 \pm 16.3$ years, with the majority being female $(56 \%)$ (Table 1). The average LOS was $7.6 \pm 9$ days (Table 2), and the median LOS was 3 days. Nearly $91 \%$ of the patients elected to be admitted. Age was neither correlated with LOS $(p=0.67)$ nor with hospital charge $(p=0.23)$. Length of stay was positively correlated with hospital charge $(r=0.78, \mathrm{p}<0.001)$.

An analysis of hospital characteristics suggested that most hospitals to which the patients with craniopharyn- 
Trends in national inpatient charges for craniopharyngioma

TABLE 1: Characteristics of the patient cohort in this study*

\begin{tabular}{lc}
\hline \multicolumn{1}{c}{ Characteristic } & Value \\
\hline age in yrs (mean \pm SD) & $47.7 \pm 16.3$ \\
sex as F/M ratio (\%) & $338: 266(56: 44)$ \\
LOS in days (mean \pm SD) & $7.6 \pm 9$ \\
no. of patients deceased & $-\dagger$ \\
admission type (no. of patients [\%]) & \\
$\quad$ elective & $549(91)$ \\
$\quad$ nonelective (urgent or ED) & $57(9)$ \\
surgical procedure (no. of patients [\%]) & \\
$\quad$ ransfrontal & $253(42)$ \\
$\quad$ transsphenoidal & $353(58)$ \\
hospital vol for procedure (no. of patients [\%]) & \\
$\quad$ performed $\leq 20$ procedures/5-yr period & $549(91)$ \\
performed $>20$ procedures/5-yr period & $57(9)$ \\
preop comorbidities (no. of patients [\%]) & \\
$\quad 0$ or 1 & $328(54)$ \\
$\geq 2$ & $278(46)$ \\
postop complications (no. of patients [\%]) & \\
$\quad 0$ & $285(47)$ \\
$\geq 1$ & $321(53)$ \\
total hospital charges in US $\$$ (mean \pm SD) & $92,300 \pm 83,356$ \\
\hline
\end{tabular}

* Unless otherwise specified, numbers and percentages represent the number of patients in the analysis. Numbers of individual patients may not add to 606 or percentages to $100 \%$ because of missing data.

† Fewer than 11 patients (2\%) were deceased.

gioma were admitted were of medium or large size (95\%), and most of these patients (84\%) had been admitted to a teaching institution. Distributions for hospital charges grouped by surgical approach, as well as by hospital and patient characteristics, are shown in Fig. 1. An analysis of the hospital charges broken down by geographical region

\section{TABLE 2: Distribution of LOS among the patients*}

\begin{tabular}{ccc}
\hline LOS (days) & No. of Patients (\%) & Cumulative Percentage (\%) \\
\hline 1 & $27(4.5)$ & 4.5 \\
2 & $84(13.9)$ & 18.3 \\
3 & $94(15.5)$ & 33.9 \\
4 & $85(14.0)$ & 47.9 \\
5 & $56(9.2)$ & 57.2 \\
6 & $55(9.1)$ & 66.3 \\
7 & $25(4.1)$ & 70.4 \\
8 & $37(6.1)$ & 76.5 \\
9 & $12(2.0)$ & 78.5 \\
10 & $15(2.5)$ & 81.0 \\
11 & $19(3.1)$ & 84.1 \\
12 & $8(1.3)$ & 85.5 \\
13 & $6(1.0)$ & 86.4 \\
14 & $7(1.2)$ & 87.6 \\
\hline
\end{tabular}

* Distribution of inpatient LOS with cumulative percentages. indicated that the Midwest had the highest total charge, whereas the South had the lowest in-hospital charges (Table 3).

\section{Complications and Hospital Charges}

Univariate analyses were used to examine the association between total in-hospital charges and complications to identify potential predictors of hospital charges in our models. Overall, 321 patients (53\%) experienced at least 1 complication. The 5 most common complications were fluid or electrolyte imbalance or diabetes insipidus (288 patients, $48 \%)$, neurological complications $(n=64$, $11 \%)$, a new cranial nerve deficit $(n=19,3 \%)$, iatrogenic panhypopituitarism $(\mathrm{n}=15,3 \%)$, and CSF rhinorrhea (n $=12,2 \%$ ). On average, patients who experienced at least 1 complication had hospital charges that were approximately double those of patients without complications $(\$ 120,863 \pm \$ 99,230$ vs $\$ 60,129 \pm \$ 41,786$, respectively; $\mathrm{p}<0.001$ ) (Table 4).

\section{Patient Acuity Covariates, Surgical Approach, and Hospital Charges}

We also examined variables related to patient acuity and surgical approach, including admission type (emergency department [ED] admission or elective surgery), comorbidities, and transfrontal versus transsphenoidal approaches. In-hospital charges for patients admitted via the ED were statistically significantly higher than those for patients admitted for elective craniopharyngioma resection $(\$ 137,693 \pm \$ 99,855$ versus $\$ 87,587 \pm \$ 80,096$, respectively; p < 0.001) (Table 4). Transfrontal procedures resulted on average in significantly higher in-hospital charges than transsphenoidal procedures $(\$ 121,542 \pm \$ 99,902$ versus $\$ 71,342 \pm \$ 61,172$, respectively; $\mathrm{p}<0.001)$.

Overall, 328 patients (54\%) had "none or 1" comorbidity (Table 1). The 5 most frequent comorbidities among our cohort were hypertension (214 patients, 35\%), hyperthyroidism $(n=143,24 \%)$, fluid electrolyte imbalance (n $=132,22 \%)$, uncomplicated diabetes $(n=102,17 \%)$, or other neurological disorders $(n=83,14 \%)$. Average hospital charges for patients with 2 or more comorbidities were significantly higher than for patients with 1 or no comorbidities $(\$ 117,715 \pm \$ 98,751$ vs $\$ 70,760 \pm \$ 59,832$, respectively; $\mathrm{p}<0.001$ ) (Table 4 ).

\section{Hospital Characteristics, Insurance Type, and Hospital Charges}

We examined several hospital characteristics, including hospital size, hospital volume, and teaching status, as well as patient insurance type, to determine those that were significantly associated with increased in-hospital charges. The mean total charges at teaching hospitals were not significantly different from charges at nonteaching hospitals $(\$ 91,866 \pm \$ 83,750$ vs $\$ 94,486 \pm \$ 73,276$, respectively; $p=0.79$ ) (Table 4 ), and charges from small hospitals were not significantly different from those at medium- or large-size hospitals $(\$ 102,168 \pm \$ 77,403$ vs $\$ 91,930 \pm \$ 82,477$, respectively; $p=0.60)$. With the exception of 2008, average hospital charges increased each year (Table 4). To examine this increase over the 5-year 
H. A. Zaidi, K. Chapple, and A. S. Little

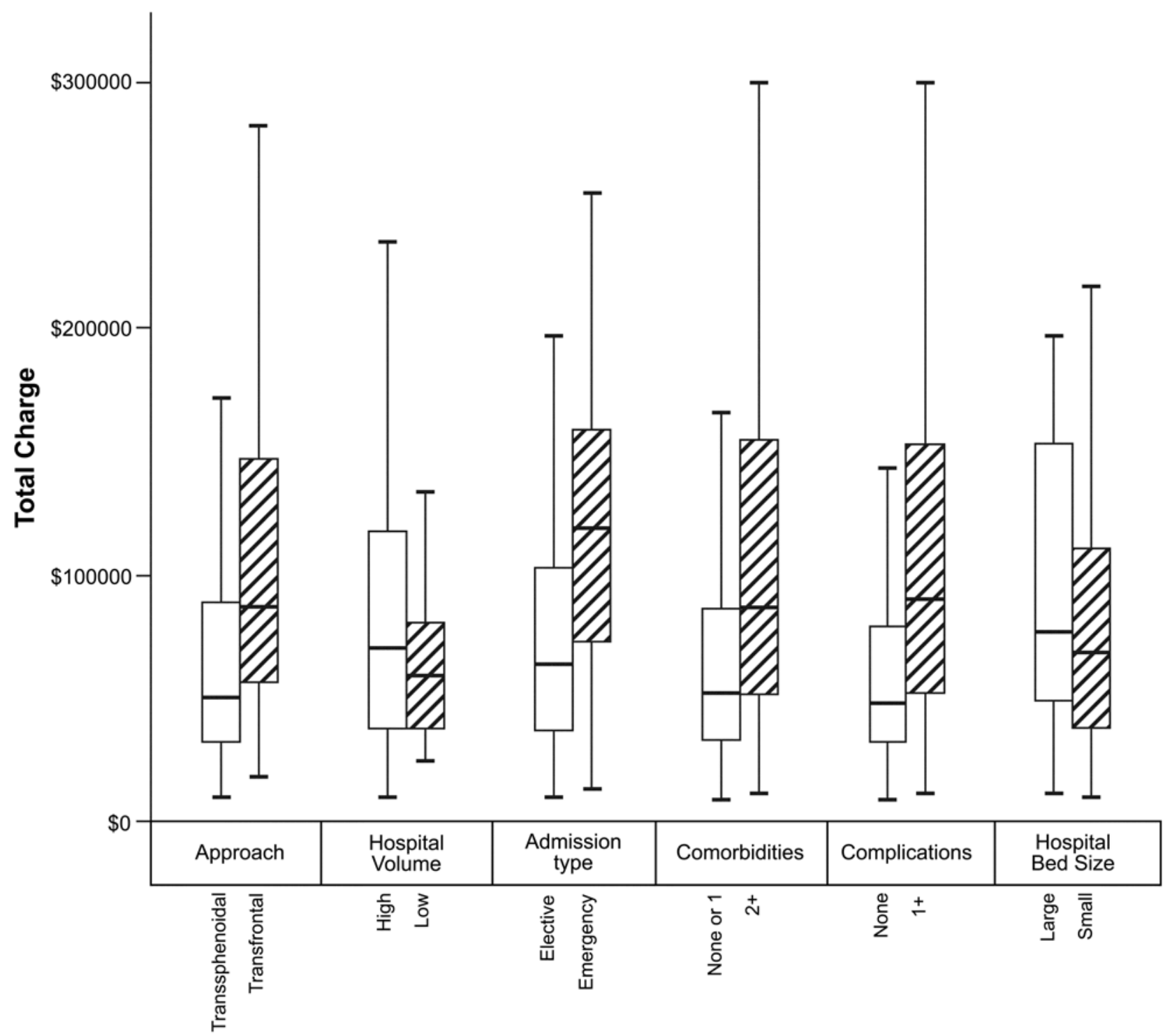

FIG. 1. Box plots indicating the median (black horizontal lines), interquartile range (open or hashed bars), and range (upper and lower error bars) of hospital charges for several key procedures, as well as hospital and patient characteristics.

study period in greater detail, an ANOVA was performed, which indicated that the year-by-year differences were statistically significant $(\mathrm{p}<0.001)$. This yearly increase in charges has been a consistent finding also for other pathologies requiring surgery ${ }^{8,29}$ and has been explained by not only the effects of inflation but also the rising cost of the delivery of health care in the United States.

Hospital volume (dichotomized by hospitals performing $\leq 20$ or $>20$ procedures in the 5 -year period) was used as a surrogate for surgeon volume because the surgeon identifier was missing in $77 \%$ of the cases. Charges associated with low-volume institutions versus high-volume institutions trended toward statistical significance $(\$ 94,358 \pm \$ 85,366$ vs $\$ 72,482 \pm \$ 57,550$, respectively; $p=0.06)$. Other factors associated with center volume were also considered and are shown in Table 5. Patients at high-volume centers had, on average, fewer complications than patients at low-volume hospitals $(0.63$ \pm 0.82 vs $1.04 \pm 0.95$, respectively; $p<0.01$ ) However, the number of hospital beds was not associated with complications $(p=0.16)$. Hospitals differing in volume showed no significant differences in average $\operatorname{LOS}(p=0.16)$ or patient age $(p=0.26)$. A review of the top 5 complication rates at high- versus low-volume hospitals revealed a statistically significant difference in the occurrence of fluid electrolyte disturbances or diabetes insipidus (35\% vs $49 \%$, respectively, $\mathrm{p}<0.05$ ) (Table 6).

Among the patients in our cohort, the majority were privately insured (57\%), followed by those who received Medicare (18\%) and Medicaid (14\%). To examine whether in-hospital charges differed by payer source, we performed an ANOVA, which detected a statistically significant difference $(\mathrm{p}<0.001)$ with highest in-hospital charges for patients with Medicaid $(\$ 138,686 \pm \$ 126,164)$, followed by Medicare $(\$ 90,198 \pm \$ 79,868)$ and private insurance $(\$ 84,132 \pm \$ 71,128)$ (Table 4). 
Trends in national inpatient charges for craniopharyngioma

TABLE 3: Hospital charges by geographical region*

\begin{tabular}{lcc}
\hline Region & No. of Patients & Hospital Charge (US\$) \\
\hline Northeast & 120 & $97,266 \pm 84,423$ \\
Midwest & 110 & $101,143 \pm 96,949$ \\
South & 154 & $80,989 \pm 84,591$ \\
West & 154 & $98,421 \pm 84,591$ \\
all $\dagger$ & 606 & $92,300 \pm 83,356$ \\
\hline
\end{tabular}

* Charges are indicated as mean $\pm \mathrm{SD}$. Northeast $=\mathrm{CT}$, MA, ME, MI, $\mathrm{NH}, \mathrm{NJ}, \mathrm{NY}, \mathrm{PA}$, and VT; Midwest = IA, IL, IN, KS, MN, MO, NE, OH, SD, and WI; South = AR, FL, GA, KY, LA, MD, NC, OK, SC, TN, TX, and VA; West = AZ, CA, CO, MT, NM, NV, OR, UT, and WA. A chi-square test comparing differences in charge among all groups resulted in a $p$ value of 0.09 .

† Also includes patients not from the 4 geographical regions above.

\section{Multivariate Regression Model}

We developed a 2-step multivariate regression model to better understand the role the aforementioned variables played in the in-hospital charges. The first step of the analysis included logarithmic transformation of the values for LOS and calendar year. In this step, with only LOS and calendar year included, our model accounted for $52 \%$ of the variation in the log-transformed total charge. In Step 2, to assess if adding other variables to the model significantly contributed to the remaining variation in the total charge, we also included characteristics of patients, procedures, and hospitals. These characteristics included hospital volume, ED admission, surgical approach, presence of 2 or more comorbidities, and the presence of 1 or more complications. The addition of these variables to our model added $2 \%$ of explained variation in the total charge.

Regression coefficients from the final step of the model are shown in Table 7. The interpretation of each predictor must be considered with all of the other predictors in the model held constant. The results of our model suggested that a $100 \%$ increase in LOS yielded a $57 \%$ increase in average total hospital charge $(\mathrm{p}<0.001)$, and each increase in calendar year was associated with an average increase of $4 \%(\mathrm{p}<0.001)$. The ED admission type and hospital size based on the number of hospital beds were not significant predictors of hospital charges. Interestingly, our multivariate model, which addressed potential confounders, indicated that high-volume hospitals were associated with an $8 \%$ increase in total charges $(\mathrm{p}<$ 0.05 ). This is in contrast to the univariate analysis of hospital volume, which suggested the opposite trend (Table $4)$. The presence of 2 or more comorbidities was associated with a $5 \%$ increase in total charges $(\mathrm{p}=0.01)$. The presence of a postoperative complication was associated with a $4 \%$ increase in total charges $(\mathrm{p}<0.05)$. Hospital charges for a transfrontal procedure were $6 \%$ higher than for a transsphenoidal procedure $(\mathrm{p}<0.01)$. An examination of the beta coefficient (B) showed that the strongest predictor of hospital charge was patient LOS, followed by high-volume hospital and by surgical approach (Table 7). Interestingly, our multivariate model, which addressed potential confounders, indicated that high-volume hospitals were associated with an $8 \%$ increase in total charges $(\mathrm{p}<0.05)$. This is in contrast to the univariate analysis of hospital volume, which suggested the opposite trend (Table 4).

\section{Discussion}

We analyzed a national administrative data set to better understand the contemporary national surgical trends and the drivers of in-hospital charges for craniopharyngioma in adults. The review included 606 adult patients surgically treated for the disease in the United States from 2007 to 2011. Our analysis demonstrated that the mean LOS of these patients was $7.6 \pm 9$ days and the inpatient mortality rate was $<2 \%$. Most patients had at least 1 postoperative complication, were treated at teaching hospitals and at low-volume centers, and underwent a transsphenoidal procedure. A multivariate model found that LOS was the most significant driver of in-hospital charges. Admission year, hospital volume, presence of preoperative comorbidities and of postoperative complications, and a transfrontal approach also predicted higher in-hospital charges, although to a lesser extent. Our findings helped identify key variables associated with in-hospital charges and are relevant to both clinicians and health care administrators. Future studies are necessary to understand what role any additional variables after discharge play in driving health care costs.

\section{Independent Predictors of In-Hospital Charges}

$L O S$. Length of stay was the most important predictor of in-hospital charges and accounted for $50 \%$ of the variance in our model. Increasing patient LOS results in higher charges for hospital rooms and for laboratory and radiological services. This finding is consistent with studies that have investigated the effect of LOS on cost of care for other neuroendocrine disorders, such as Cushing disease. ${ }^{22,27}$ For example, Patil et al. noted that a single postoperative complication would increase LOS by 3 days and increase hospital charges by more than $\$ 7000 .{ }^{27}$ Similarly, our group recently found that a $1 \%$ increase in LOS after surgery for Cushing disease resulted in a $0.6 \%$ increase in hospital charges. ${ }^{22}$ From a practical standpoint, this is an important finding for clinicians managing patients after craniopharyngioma surgery. It indicates that efforts to streamline the discharge process can potentially have one of the highest impacts on reducing in-hospital charges. Aggressively managing postoperative pain, setting patients' expectations on LOS in the clinic after surgery, discharging those with uncomplicated resections early, and managing electrolyte imbalance in outpatient settings may reduce hospital charges indirectly by reducing LOS. Furthermore, the implementation of medical checklists has been shown to decrease LOS and may be useful in this setting. ${ }^{19}$

Institutional Volume. Our model also revealed that surgeries at high-volume hospitals were associated with lower rates of perioperative complications than those 
H. A. Zaidi, K. Chapple, and A. S. Little

TABLE 4: Average hospital charges by hospital or patient characteristic*

\begin{tabular}{|c|c|c|c|}
\hline Characteristic & Cost (US\$) & $\mathrm{p}$ Value & Partial Eta-Squared Effect Size \\
\hline procedure & & $<0.001$ & 0.09 \\
\hline transfrontal & $121,542 \pm 99,902$ & & \\
\hline transsphenoidal & $71,342 \pm 61,172$ & & \\
\hline admission type & & $<0.001$ & 0.03 \\
\hline ED & $137,693 \pm 99,855$ & & \\
\hline routine & $87,587 \pm 80,096$ & & \\
\hline hospital type & & 0.79 & 0.00 \\
\hline teaching & $91,866 \pm 83,750$ & & \\
\hline nonteaching & $94,486 \pm 73,276$ & & \\
\hline hospital procedures over 5 yrs & & 0.06 & 0.01 \\
\hline$>20$ procedures & $72,482 \pm 57,550$ & & \\
\hline$\leq 20$ procedures & $94,358 \pm 85,366$ & & \\
\hline hospital size & & 0.60 & 0.00 \\
\hline small & $102,168 \pm 77,403$ & & \\
\hline medium/large & $91,930 \pm 82,477$ & & \\
\hline postop complications & & $<0.001$ & 0.13 \\
\hline complications & $120,863 \pm 99,230$ & & \\
\hline no complications & $60,129 \pm 41,786$ & & \\
\hline preop comorbidities & & $<0.001$ & 0.08 \\
\hline 0 or 1 & $70,760 \pm 59,832$ & & \\
\hline$\geq 2$ & $117,715 \pm 98,751$ & & \\
\hline payer source & & $<0.001$ & 0.05 \\
\hline private insurance & $84,132 \pm 71,128$ & & \\
\hline Medicare & $90,198 \pm 79,868$ & & \\
\hline Medicaid & $138,686 \pm 126,164$ & & \\
\hline self-pay & $81,915 \pm 70,257$ & & \\
\hline other & $76,424 \pm 50,591$ & & \\
\hline calendar yr & & $<0.001$ & 0.04 \\
\hline 2007 & $80,547 \pm 86,916$ & & \\
\hline 2008 & $74,556 \pm 67,358$ & & \\
\hline 2009 & $84,199 \pm 68,576$ & & \\
\hline 2010 & $97,880 \pm 86,082$ & & \\
\hline 2011 & $116,791 \pm 96,259$ & & \\
\hline
\end{tabular}

* Costs are indicated as mean \pm SD. The $p$ values indicate results of an ANOVA.

performed at low-volume ones. Previous reports from various subspecialties indicate that the annual number of a specific surgical procedure a surgeon performs directly correlates with improved health-related outcomes, ${ }^{1-3,7,11,12,15}$ and our group recently demonstrated similar findings for Cushing disease. ${ }^{22}$ In support of this finding, the incidence of the most common complication, fluid electrolyte imbalance or diabetes, was significantly lower in high-volume centers. One potential explanation may be that experienced surgeons are considerably better at identifying and preserving the pituitary stalk and limiting trauma to the hypothalamus. A limitation of our study is that the administrative database does not contain any tumor-specific information (for example, size, local brain invasion, and location). We were therefore unable to normalize our data for case complexity between centers. In-
TABLE 5: Characteristics of patients treated at high- or low-volume hospitals*

\begin{tabular}{|c|c|c|c|}
\hline Variable & $\begin{array}{l}\text { High-Vol } \\
\text { Hospital }\end{array}$ & $\begin{array}{l}\text { Low-Vol } \\
\text { Hospital }\end{array}$ & p Value $†$ \\
\hline LOS (days) & $5.9 \pm 7.3$ & $7.7 \pm 9.2$ & 0.16 \\
\hline no. of complications & $0.6 \pm 0.8$ & $1.0 \pm 0.9$ & $<0.01$ \\
\hline age at admission (yrs) & $45.4 \pm 16.2$ & $48.0 \pm 16.3$ & 0.26 \\
\hline
\end{tabular}


Trends in national inpatient charges for craniopharyngioma

TABLE 6: Comparison of the types of complications in patients treated at high- versus low-volume hospitals*

\begin{tabular}{lccc}
\hline \multirow{2}{*}{\multicolumn{1}{c}{ Complication }} & \multicolumn{2}{c}{ No. of Patients (\%) } & \\
\cline { 2 - 3 } & $\begin{array}{c}\text { High-Vol } \\
\text { Hospitals }\end{array}$ & $\begin{array}{c}\text { Low-Vol } \\
\text { Hospitals }\end{array}$ & p Value† \\
\hline $\begin{array}{l}\text { fluid electrolyte imbalance/ } \\
\quad \text { diabetes insipidus }\end{array}$ & $20(35)$ & $268(49)$ & 0.048 \\
neurological complications & $7(12)$ & $57(10)$ & 0.65 \\
cranial nerve complication & $-\ddagger$ & $18(3)$ & 0.53 \\
iatrogenic panhypopituitarism & $-\ddagger$ & $15(3)$ & 0.21 \\
CSF rhinorrhea & $-\ddagger$ & $11(2)$ & 0.89 \\
\hline
\end{tabular}

* Hospital volume was defined as described in first footnote of Table 5; percentages in each column were calculated with the total number of patients at high- and low-volume hospitals (57 and 549 patients, respectively; see Table 1).

$\dagger$ The $p$ values indicate results of chi-square tests.

$\ddagger$ Indicates that fewer than 11 patients or $<2 \%$ of patients showed this complication.

terestingly, we found that the lower risk for complications at high-volume centers did not translate into a shorter LOS. One explanation may be that efficient postoperative care for this cohort at high-volume centers is offset by a higher complexity of the craniopharyngioma cases (with a longer postoperative course and rehabilitation) referred to these centers.

Counterintuitively, in our univariate model, we found that patients with craniopharyngiomas treated at highvolume centers had on average a lower complication rate; however, in our multivariate model, with other predictors held constant, high-volume centers were associated with an $8 \%$ increase in hospital charges. One possible explanation for this apparent contradiction is that the use of expensive surgical disposables, sophisticated diagnostic tools (3-T and intraoperative MRI), and a highly multidisciplinary approach to patient care (that is, neurosurgery, neurology, pathology, neuroendocrinology, or neurocritical care) at these high-volume tertiary care centers may contribute to higher overall charges. These findings complicate the recent debate calling for high-volume centers of excellence in pituitary surgery. ${ }^{30}$ Advocates for such centers have focused on surgical outcomes, and the full financial impact of using such centers has not been taken into account. A more comprehensive, prospectively designed, cost-benefit analysis is necessary to better understand not only the clinical but also the financial impact of frequently referring complex pathologies of pituitary regions to highly specialized tertiary centers. This type of study could assess whether our observation is sound, or whether an unmeasured confounding variable affected our current analysis. Of important note, because of the high number of missing surgeon identifiers in the NIS data set, we could not determine how many surgeons at a particular institution were managing craniopharyngioma patients and what their level of experience was.

Preoperative Morbidities. Our multivariate analysis indicated that patients with preoperative morbidities had significantly higher in-hospital charges. This finding was expected considering that these patients may require additional management of their comorbid conditions, which perhaps increases LOS. Several groups have previously demonstrated that improving comorbid conditions presurgery increases postoperative survival and decreases tumor recurrence and functional loss after surgery for gliomas. ${ }^{5,21,23,34}$ Here, we demonstrated that the presence of comorbid conditions preoperatively was associated with higher overall in-hospital charge. Appropriate patient selection with mitigation of medical issues prior to surgery may play a more important role in in-hospital resource use than previously assumed. Therefore, preoperative morbidity may be an important modifiable factor to target in order to reduce in-hospital charges.

Admission Source. Admission from the ED was an independent predictor of increased in-hospital charges, and this may be explained by 1 of 2 scenarios. Patients admitted via the ED may require increased resource utilization during the in-hospital admission. These resources include preoperative diagnostic workups, such as imaging and laboratory analyses, which normally would have been performed in an outpatient setting for elective surgery. Alternatively, patients who present to the ED may have greater disease severity. Unfortunately, the NIS data set does not provide information on disease severity, so we are unable to address this point.

TABLE 7: Linear regression coefficients predicting total hospital charges*

\begin{tabular}{lccccc}
\hline \multicolumn{1}{c}{ Predictor } & B & SE B & LCL & UCL & p Value \\
\hline LOS (log transformed) & 0.57 & 0.03 & 0.51 & 0.63 & $<0.001$ \\
yr of surgery & 0.04 & 0.01 & 0.03 & 0.05 & $<0.001$ \\
ED admission & -0.03 & 0.03 & -0.09 & 0.04 & 0.43 \\
hospital size (medium to large) & -0.04 & 0.05 & -0.14 & 0.06 & 0.47 \\
high-vol hospital & 0.08 & 0.03 & 0.01 & 0.14 & 0.02 \\
comorbidities ( $\geq 2)$ & 0.05 & 0.02 & 0.01 & 0.09 & 0.01 \\
complications ( $\geq 1)$ & 0.04 & 0.02 & 0.00 & 0.08 & 0.04 \\
transfrontal approach & 0.06 & 0.02 & 0.02 & 0.10 & 0.01 \\
\hline
\end{tabular}

* The coefficients listed predicted total hospital charges that were log-transformed for the regression analysis. $B=$ beta-coefficient; LCL = lower confidence limit; SE B = standardized beta coefficient; UCL = upper confidence limit. 
Surgical Approaches. A transfrontal approach in craniopharyngioma resection was an independent predictor of higher in-hospital charges, and hospital charges for a transfrontal procedure were $6 \%$ higher than for a transsphenoidal procedure. The NIS data set does not include tumor-specific data, such as tumor size and configuration, or details regarding surgical strategy, such as the application of an endoscopic or microscopic technique, or attempted gross-total resection or internal debulking. Therefore, we could not determine whether transsphenoidal surgery was significantly associated with lower charges compared with a transfrontal approach. We were also unable to compare the impact on charge of planned subtotal resection with that of attempted gross-total resection. We hypothesize that the difference in charges between these 2 approaches may be explained by the fact that more challenging tumors are addressed through a transfrontal approach. Further prospective studies are necessary to assess the root cause of these differences in in-hospital charge.

\section{Study Limitations}

Our study methods were limited by the NIS data set and the data types available for the analysis. First, administrative databases are largely compiled by coders who are not clinicians, and any miscoding may have complicated the analysis. Both Barker et al. and Patil et al. noted that the reported rates of several variables in the NIS database differed from those in institutional databases. ${ }^{2,27}$ Second, the NIS database does not provide charge data for readmissions and outpatient care. This is an important limitation in the analysis of patients with a high risk for readmission and significant use of outpatient resources as is the case for patients with craniopharyngioma. Clearly, a study of long-term resource utilization is an important next step that may be possible by using other administrative data sets or a collaborative multicenter effort. Third, the NIS data set reports hospital charges rather than direct or indirect (for example, overhead) costs of providing care. While hospital charges are an established method for studying resource use, they have a variable relationship to costs. Finally, our analysis, as retrospective analyses are in general, was limited to identifying associations between the variables, which does not necessarily imply that these associations were the result of causative relationships.

\section{Conclusions}

Craniopharyngiomas present surgical challenges that necessitate a multidisciplinary approach. Until now, national treatment trends and in-hospital charges for this disease have not been well studied. The data presented here provide a snapshot of rates of inpatient mortality and complications, as well as of the characteristics of the patients with craniopharyngioma. After accounting for demographic information, patient acuity variables, hospital characteristics, and patient complications, we found LOS, high institutional volume, increased postoperative complications, calendar year, and increased preoperative comorbidities to be significantly associated with higher in-hospital charges. Identifying the drivers of inpatient resource use may help improve future efforts to optimize resource utilization. Studies evaluating the long-term resource utilization in patients undergoing treatment for craniopharyngioma or for diseases of similar severity would provide useful future directions.

\section{Acknowledgments}

We thank the Barrow Neurological Institute for providing the funding that supported this study. We also wish to thank the Neuroscience Publications office at Barrow Neurological Institute for assistance with the preparation of this manuscript.

\section{Disclosure}

The authors report no conflicts of interests with any of the material or methods in this study.

Author contributions to the study and manuscript preparation include the following. Conception and design: Little, Zaidi. Acquisition of data: Little, Chapple. Analysis and interpretation of data: all authors. Drafting the article: Zaidi. Critically revising the article: all authors. Reviewed submitted version of manuscript: all authors. Approved the final version of the manuscript on behalf of all authors: Little. Statistical analysis: Chapple. Study supervision: Little.

\section{References}

1. Archampong D, Borowski D, Wille-Jørgensen P, Iversen LH: Workload and surgeon's specialty for outcome after colorectal cancer surgery. Cochrane Database Syst Rev 3:CD005391, 2012

2. Barker FG II, Klibanski A, Swearingen B: Transsphenoidal surgery for pituitary tumors in the United States, 1996-2000: mortality, morbidity, and the effects of hospital and surgeon volume. J Clin Endocrinol Metab 88:4709-4719, 2003

3. Begg CB, Cramer LD, Hoskins WJ, Brennan MF: Impact of hospital volume on operative mortality for major cancer surgery. JAMA 280:1747-1751, 1998

4. Bunin GR, Surawicz TS, Witman PA, Preston-Martin S, Davis F, Bruner JM: The descriptive epidemiology of craniopharyngioma. J Neurosurg 89:547-551, 1998

5. Chaichana KL, McGirt MJ, Woodworth GF, Datoo G, Tamargo RJ, Weingart J, et al: Persistent outpatient hyperglycemia is independently associated with survival, recurrence and malignant degeneration following surgery for hemispheric low grade gliomas. Neurol Res 32:442-448, 2010

6. Chiou SM, Lunsford LD, Niranjan A, Kondziolka D, Flickinger JC: Stereotactic radiosurgery of residual or recurrent craniopharyngioma, after surgery, with or without radiation therapy. Neuro Oncol 3:159-166, 2001

7. Chiu CC, Wang JJ, Tsai TC, Chu CC, Shi HY: The relationship between volume and outcome after bariatric surgery: a nationwide study in Taiwan. Obes Surg 22:1008-1015, 2012

8. Daniels AH, Arthur M, Esmende SM, Vigneswaran H, Palumbo MA: Incidence and cost of treating axis fractures in the United States from 2000 to 2010. Spine (Phila Pa 1976) 39:1498-1505, 2014

9. Epstein NE: A review of interspinous fusion devices: high complication, reoperation rates, and costs with poor outcomes. Surg Neurol Int 3:7, 2012

10. Epstein NE, Schwall G, Reillly T, Insinna T, Bahnken A, Hood DC: Surgeon choices, and the choice of surgeons, affect total hospital charges for single-level anterior cervical surgery. Spine (Phila Pa 1976) 36:905-909, 2011

11. Gourin CG, Frick KD: National trends in oropharyngeal cancer surgery and the effect of surgeon and hospital volume on shortterm outcomes and cost of care. Laryngoscope 122:543-551, 2012 


\section{Trends in national inpatient charges for craniopharyngioma}

12. Hillner BE, Smith TJ, Desch CE: Hospital and physician volume or specialization and outcomes in cancer treatment: importance in quality of cancer care. J Clin Oncol 18:2327-2340, 2000

13. Hoh BL, Chi YY, Dermott MA, Lipori PJ, Lewis SB: The effect of coiling versus clipping of ruptured and unruptured cerebral aneurysms on length of stay, hospital cost, hospital reimbursement, and surgeon reimbursement at the university of Florida. Neurosurgery 64:614-621, 2009

14. Hoh BL, Chi YY, Lawson MF, Mocco J, Barker FG II: Length of stay and total hospital charges of clipping versus coiling for ruptured and unruptured adult cerebral aneurysms in the Nationwide Inpatient Sample database 2002 to 2006. Stroke 41:337-342, 2010 (Erratum in Stroke 42:e356, 2011)

15. Hornik CP, He X, Jacobs JP, Li JS, Jaquiss RD, Jacobs ML, et al: Relative impact of surgeon and center volume on early mortality after the Norwood operation. Ann Thorac Surg 93: 1992-1997, 2012

16. Houchens RL, Elixhauser A: Using the HCUP Nationwide Inpatient Sample to estimate trends (updated for 1988-2004). HCUP Methods Series Report \#2006-05. Healthcare Cost and Utilization Project. (http://www.hcup-us.ahrq.gov/re ports/methods/2006_05_NISTrendsReport_1988-2004.pdf) [Accessed September 14, 2014]

17. Jeon C, Kim S, Shin HJ, Nam DH, Lee JI, Park K, et al: The therapeutic efficacy of fractionated radiotherapy and gammaknife radiosurgery for craniopharyngiomas. J Clin Neurosci 18:1621-1625, 2011

18. Karavitaki N, Brufani C, Warner JT, Adams CB, Richards P, Ansorge O, et al: Craniopharyngiomas in children and adults: systematic analysis of 121 cases with long-term follow-up. Clin Endocrinol (Oxf) 62:397-409, 2005

19. Lepänluoma M, Takala R, Kotkansalo A, Rahi M, Ikonen TS: Surgical safety checklist is associated with improved operating room safety culture, reduced wound complications and unplanned readmissions in a pilot study in neurosurgery. Scand J Surg 103:66-72, 2014

20. Levin DA, Bendo JA, Quirno M, Errico T, Goldstein J, Spivak $\mathrm{J}$ : Comparative charge analysis of one- and two-level lumbar total disc arthroplasty versus circumferential lumbar fusion. Spine (Phila Pa 1976) 32:2905-2909, 2007

21. Link TW, Woodworth GF, Chaichana KL, Grossman SA, Mayer RS, Brem H, et al: Hyperglycemia is independently associated with post-operative function loss in patients with primary eloquent glioblastoma. J Clin Neurosci 19:996-1000, 2012

22. Little AS, Chapple K: Predictors of resource utilization in transsphenoidal surgery for Cushing disease. Clinical article. J Neurosurg 119:504-511, 2013

23. McGirt MJ, Chaichana KL, Gathinji M, Attenello F, Than K, Ruiz AJ, et al: Persistent outpatient hyperglycemia is independently associated with decreased survival after primary resection of malignant brain astrocytomas. Neurosurgery 63: 286-291, 2008
24. Mortini P, Gagliardi F, Boari N, Losa M: Surgical strategies and modern therapeutic options in the treatment of craniopharyngiomas. Crit Rev Oncol Hematol 88:514-529, 2013

25. Mukherjee D, Zaidi HA, Kosztowski T, Chaichana KL, Salvatori R, Chang DC, et al: Predictors of access to pituitary tumor resection in the United States, 1988-2005. Eur J Endocrinol 161:259-265, 2009

26. Mukherjee D, Zaidi HA, Kosztowski TA, Halthore A, Jallo GI, Salvatori R, et al: Variations in referral patterns for hypophysectomies among pediatric patients with sellar and parasellar tumors. Childs Nerv Syst 26:305-311, 2010

27. Patil CG, Lad SP, Harsh GR, Laws ER Jr, Boakye M: National trends, complications, and outcomes following transsphenoidal surgery for Cushing's disease from 1993 to 2002. Neurosurg Focus 23(3):E7, 2007

28. Rodriguez FJ, Scheithauer BW, Tsunoda S, Kovacs K, Vidal S, Piepgras DG: The spectrum of malignancy in craniopharyngioma. Am J Surg Pathol 31:1020-1028, 2007

29. Rosenbaum BP, Kelly ML, Kshettry VR, Vadera S, Weil RJ: Practice patterns of in-hospital surgical treatment of trigeminal neuralgia from 1988 to 2010. Clin Neurol Neurosurg 120:55-63, 2014

30. Schwartz TH: A role for centers of excellence in transsphenoidal surgery. World Neurosurg 80:270-271, 2013

31. Tomita T, Bowman RM: Craniopharyngiomas in children: surgical experience at Children's Memorial Hospital. Childs Nerv Syst 21:729-746, 2005

32. Wang MY, Cummock MD, Yu Y, Trivedi RA: An analysis of the differences in the acute hospitalization charges following minimally invasive versus open posterior lumbar interbody fusion. Clinical article. J Neurosurg Spine 12:694-699, 2010

33. Whitmore RG, Schwartz JS, Simmons S, Stein SC, Ghogawala Z: Performing a cost analysis in spine outcomes research: comparing ventral and dorsal approaches for cervical spondylotic myelopathy. Neurosurgery 70:860-867, 2012

34. Woodworth GF, Chaichana KL, McGirt MJ, Sciubba DM, Jallo GI, Gokaslan Z, et al: Predictors of ambulatory function after surgical resection of intramedullary spinal cord tumors. Neurosurgery 61:99-106, 2007

Manuscript submitted June 18, 2014.

Accepted August 25, 2014.

Please include this information when citing this paper: DOI: 10.3171/2014.8.FOCUS14366.

Address correspondence to: Andrew S. Little, M.D., Neuroscience Publications, Barrow Neurological Institute, St. Joseph's Hospital and Medical Center, 350 W. Thomas Rd., Phoenix, AZ 85013. email: neuropub@dignityhealth.org. 